\title{
Cepheus Star-Forming Field Revisited
}

\author{
Nadia Kaltcheva $^{1}$, Vincent Fabbri ${ }^{1}$, Timothy Conard ${ }^{1}$, Valeri Golev ${ }^{2}$ \\ ${ }^{1}$ Department of Physics and Astronomy, University of Wisconsin, Oshkosh, USA \\ ${ }^{2}$ Department of Astronomy, Faculty of Physics, St Kliment Ohridski University of Sofia, \\ Sofia, Bulgaria \\ Email: kaltchev@uwosh.edu, valgol@phys.uni-sofia.bg
}

Received October 10, 2013; revised November 5, 2013; accepted November 12, 2013

Copyright (C) 2013 Nadia Kaltcheva et al. This is an open access article distributed under the Creative Commons Attribution License, which permits unrestricted use, distribution, and reproduction in any medium, provided the original work is properly cited.

\begin{abstract}
We present a photometric investigation in the $u v b y \beta$ system of a $20^{\circ} \times 20^{\circ}$ field centered at galactic coordinates $(1, \mathrm{~b})=$ $\left(105^{\circ}, 5^{\circ}\right)$. This region contains a relatively isolated structure of numerous HII regions in the direction of some of the OB associations in Cepheus and Cassiopeia. Photoelectric $u v b y \beta$ data of more than 220 stars of spectral types O-B9 are available for this field and are used to obtain homogeneous distance estimates for a number of prominent stellar groups, among which are the Cep OB2 and Cep OB3 associations and the open clusters NGC 7243 and NGC 7654.
\end{abstract}

Keywords: Open Clusters and Associations: Individual (Cep OB2); Open Clusters and Associations: Individual (Cep OB3); Open Clusters and Associations: Individual (NGC 7243); Open Clusters and Associations: Individual (NGC 7654)

\section{Introduction}

The field between $95^{\circ}$ and $116^{\circ}$ galactic longitude and $-5^{\circ}$ and $15^{\circ}$ galactic latitude contains numerous HII regions. Among them Sh 2-131, Sh 2-132 and Sh 2-157 are the most prominent, associated with some very young open clusters. Several OB associations have been identified in this direction (Cep OB1, Cep OB2, Cep OB3, Cep OB5 and Cas OB2), some of which are well studied in terms of broad-band photometry (see Humphreys [1] and the references therein).

The goal of this investigation is to provide homogeneous distance and reddening estimates for as many young stars as possible in the studied coordinate range. We use the intermediate-band $u v b y \beta$ photometric system (Strömgren [2], Crawford \& Mander [3]), since it allows reliable distance determination which helps distinguish among closely spaced groups along the line of sight (see for example Kaltcheva \& Golev [4]). This study is aimed to provide the basis for a further detailed investigation of the interaction between the OB stars and the interstellar medium for this field.

\section{The Sample}

The field is selected to cover the isolated large-scale feature seen in $\mathrm{H} \alpha$ between $95^{\circ}$ and $116^{\circ}$ galactic longitude. All $u v b y \beta$ data within this coordinate range was extracted from the catalog of Hauck \& Mermilliod [5]. The sample contains 222 stars of spectral types O to B9 with complete $u v b y \beta$ photometry. The sample stars are presented in Figure 1. They are overplotted on an $\mathrm{H} \alpha$ map smoothed to 4 arcmin resolution to remove star residuals, Gaustad et al. [6], which is obtained via the SkyView VO interface (McGlynn et al. [7]).

\section{Calculation of Interstellar Extinction and Stellar Distances}

The color excess $\mathrm{E}(\mathrm{b}-\mathrm{y})$ for stars of luminosity classes (LC) III, IV and V was obtained via the Crawford's [8] calibration. The calibration by Kilkenny \& Whittet [9] was used for LC II, Ib, Iab and Ia. We used $\mathrm{R}=3.18$ and $\mathrm{E}(\mathrm{B}-\mathrm{V})=\mathrm{E}(\mathrm{b}-\mathrm{y}) / 0.74$ to obtain the corrected for reddening visual magnitude $\mathrm{V}_{0}$. The calibration by Balona \& Shobbrook [10] was applied to derive the absolute magnitude $\mathrm{M}_{\mathrm{V}}$ for each star. For stars indicated as emission-line stars, $\beta$ calculated from $\mathrm{c}_{0}$ was used to calculate $\mathrm{M}_{\mathrm{V}}$. This procedure has been described in detail in earlier studies of star-forming fields based on $u v b y \beta$ photometry (see for example Kaltcheva \& Hilditch [11]). Note that the $u v b y \beta$ photometric distances derived in this way are in excellent agreement with the recalculated Hipparcos data (Kaltcheva \& Makarov [12]). In addition, the by Balona \& Shobbrook [10] luminosity calibration has been 


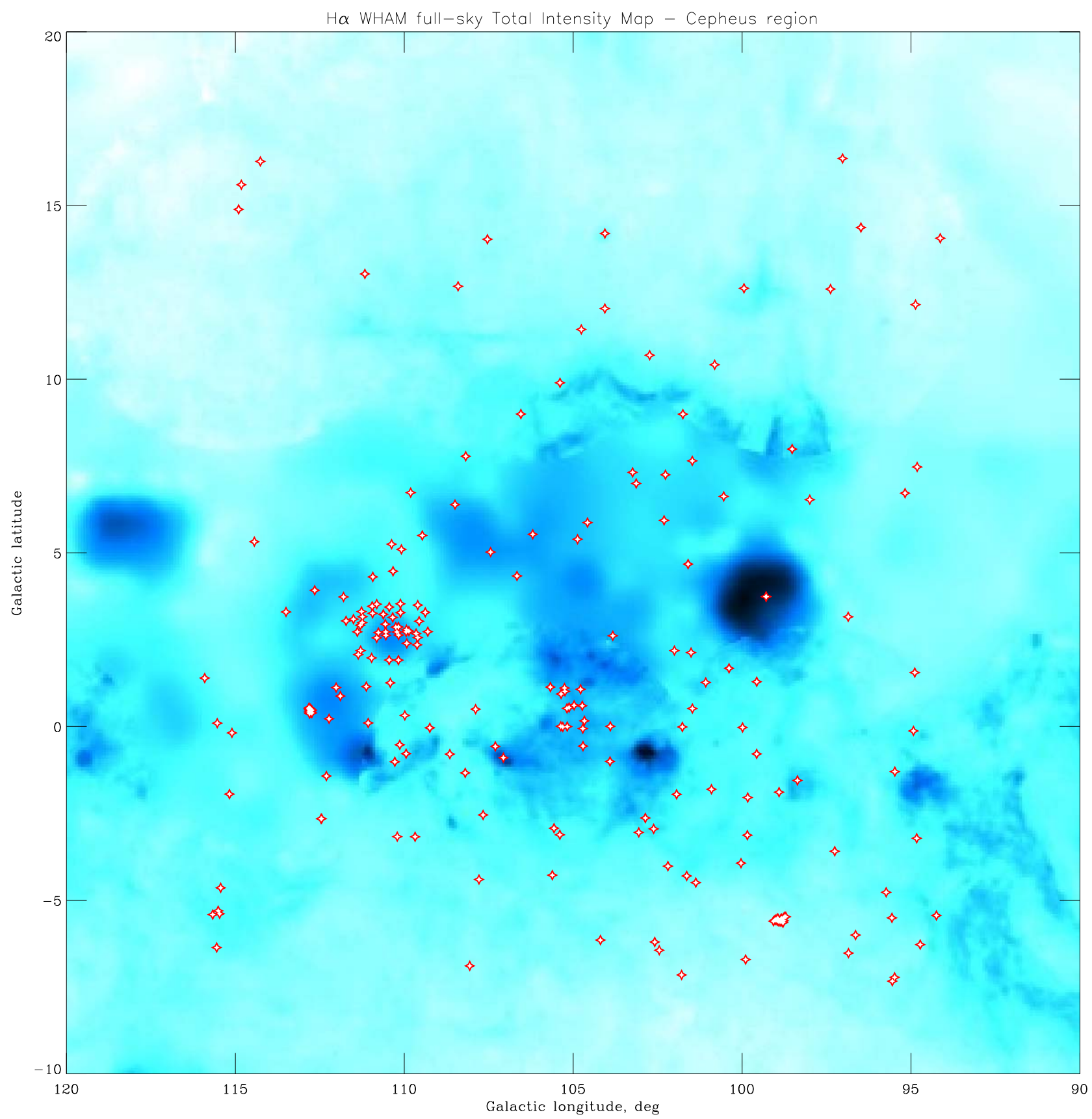

Figure 1. All stars in the studied field with uvby $\beta$ photometry available in the Hauck \& Mermilliod catalog [5] plotted in galactic coordinates. The stars are superimposed on a Ha map obtained via the SkyView VO interface (see text for details).

tested via the Hipparcos data and shown to be reliable (Kaltcheva \& Knude [13], Torra et al. [14], Kaltcheva \& Golev [15]). Note that in the $u v b y \beta$ system both the color excess and absolute magnitude calculations do not rely on a precise determination of spectral type and, to some extent, the luminosity class. This is because the calculations are carried on in the same way for all stars in a given spectral range (e.g. O-B9), depending on the LC for the color excess derivation only. The spectral and luminosity classes used in these calculations were ex- tracted from the SIMBAD database and verified based on the classification $\left[\mathrm{c}_{1}\right]$ vs. $\left[\mathrm{m}_{1}\right]$ diagram (Strömgren [2] not shown here). The expected uncertainties in the derived distances are of the order of $-13 \%$ to $+15 \%$ for $\mathrm{LC}$ III-V, and $-21 \%$ to $+26 \%$ for LC I-II.

Table 1 (available in full from the authors upon request) summarizes the photometric data and the derived stellar parameters. The table includes stellar identifications, followed by spectral and luminosity type and $u v b y \beta$ photometric data, color excess and dereddened 
Table 1. Distance modulus and color excess for the clusters and OB associations represented in the sample.

\begin{tabular}{|c|c|c|c|c|c|c|c|c|c|c|c|c|c|c|}
\hline LID & V & $b-y$ & $\mathrm{~m}_{1}$ & $\mathrm{c}_{1}$ & $\beta$ & $\mathrm{Sp}, \mathrm{LC}$ & $(b-y)_{0}$ & $\mathrm{c}_{0}$ & $\mathrm{~m}_{0}$ & $\mathrm{E}(\mathrm{b}-\mathrm{y})$ & $\mathrm{V}_{0}$ & $\mathrm{Mv}$ & DM & Notes \\
\hline 900020004 & 10.78 & 0.297 & 0.028 & 1.051 & 2.862 & B5 & -0.02 & 0.991 & 0.133 & 0.317 & 9.416 & 0.89 & 8.53 & Cep OB3 FG \\
\hline 900020034 & 10.6 & 0.275 & 0.039 & 0.986 & 2.845 & A1V & -0.026 & 0.929 & 0.138 & 0.301 & 9.305 & 0.75 & 8.56 & Cep OB3 FG \\
\hline 900020064 & 9.58 & 0.236 & 0.067 & 1.208 & 2.826 & A1V & -0.003 & 1.163 & 0.146 & 0.239 & 8.55 & 0.25 & 8.30 & Cep OB3 FG \\
\hline 900020065 & 10.14 & 0.196 & 0.06 & 0.946 & 2.816 & B9V & -0.029 & 0.903 & 0.134 & 0.225 & 9.174 & 0.43 & 8.75 & Cep OB3 FG \\
\hline 900020055 & 9.99 & 0.223 & 0.063 & 1.055 & 2.803 & $\mathrm{~A} 0 \mathrm{~V}$ & -0.018 & 1.009 & 0.143 & 0.241 & 8.952 & 0.13 & 8.82 & Cep OB3 FG \\
\hline 900020072 & 8.15 & 0.233 & -0.044 & 0.875 & 2.718 & B8III & -0.036 & 0.824 & 0.045 & 0.269 & 6.992 & -1.10 & 8.09 & Cep OB3 FG \\
\hline 900020078 & 8.58 & 0.087 & 0.075 & 0.939 & 2.747 & B9Vn & -0.027 & 0.917 & 0.113 & 0.114 & 8.089 & -0.67 & 8.76 & Cep OB3 FG \\
\hline 900020082 & 9.35 & 0.083 & 0.105 & 0.942 & 2.837 & B9.5V & -0.027 & 0.921 & 0.141 & 0.11 & 8.878 & 0.66 & 8.21 & Cep OB3 FG \\
\hline 900020074 & 10.56 & 0.153 & 0.096 & 1.049 & 2.86 & A3V & -0.018 & 1.017 & 0.152 & 0.171 & 9.827 & 0.85 & 8.98 & Cep OB3 FG \\
\hline 900020002 & 9.23 & 0.605 & -0.178 & 0.149 & 2.626 & B1V & -0.116 & 0.012 & 0.06 & 0.721 & 6.132 & -2.95 & 9.08 & Cep OB3 \\
\hline 6202114 & 9.61 & 0.379 & -0.068 & 0.107 & 2.665 & B8 & -0.115 & 0.013 & 0.095 & 0.494 & 7.485 & -2.29 & 9.77 & Cep OB3 \\
\hline 900020016 & 8.92 & 0.562 & -0.104 & 0.05 & 2.611 & - & -0.124 & -0.08 & 0.123 & 0.686 & 5.968 & -3.76 & 9.73 & Cep OB3 \\
\hline 900020010 & 8.03 & 0.48 & -0.141 & 0.029 & 2.613 & $08.5 \mathrm{~V}$ & -0.125 & -0.086 & 0.059 & 0.605 & 5.429 & -3.75 & 9.18 & Cep OB3 \\
\hline 900020020 & 9.11 & 0.513 & -0.124 & 0.111 & 2.629 & B1V & -0.117 & -0.009 & 0.084 & 0.63 & 6.399 & -2.99 & 9.38 & Cep OB3 \\
\hline 900020031 & 8.01 & 0.46 & -0.12 & 0.004 & 2.611 & $08.5 \mathrm{~V}$ & -0.127 & -0.108 & 0.074 & 0.587 & 5.486 & -3.95 & 9.44 & Cep OB3 \\
\hline 900020022 & 9.24 & 0.436 & -0.096 & 0.055 & 2.622 & B1V & -0.121 & -0.051 & 0.088 & 0.557 & 6.843 & -3.35 & 10.19 & Cep OB3 \\
\hline 900020024 & 9.71 & 0.604 & -0.173 & 0.129 & 2.644 & B1V & -0.117 & -0.008 & 0.065 & 0.721 & 6.608 & -2.71 & 9.32 & Cep OB3 \\
\hline 900020019 & 10.4 & 0.318 & 0.014 & 0.387 & 2.715 & B7V & -0.086 & 0.31 & 0.147 & 0.404 & 8.661 & -0.81 & 9.48 & Cep OB3 \\
\hline 900020014 & 9.03 & 0.532 & -0.117 & 0.166 & 2.635 & $\mathrm{~B} 1.5 \mathrm{~V}$ & -0.112 & 0.044 & 0.096 & 0.644 & 6.259 & -2.65 & 8.91 & Cep OB3 \\
\hline 900020040 & 8.8 & 0.556 & -0.109 & 0.112 & 2.632 & $\mathrm{~B} 1 \mathrm{Vn}$ & -0.118 & -0.016 & 0.113 & 0.674 & 5.901 & -2.96 & 8.86 & Cep OB3 \\
\hline 900020041 & 7.71 & 0.532 & -0.156 & -0.002 & 2.596 & - & -0.129 & -0.128 & 0.062 & 0.661 & 4.868 & -4.47 & 9.34 & Cep OB3 \\
\hline 900020039 & 7.74 & 0.424 & -0.117 & 0.006 & 2.617 & - & -0.126 & -0.099 & 0.065 & 0.55 & 5.375 & -3.76 & 9.13 & Cep OB3 \\
\hline 900020037 & 10.95 & 0.438 & -0.028 & 0.597 & 2.727 & B6Vnnp & -0.068 & 0.501 & 0.139 & 0.506 & 8.775 & -0.56 & 9.34 & Cep OB3 \\
\hline 900020054 & 9.02 & 0.43 & -0.07 & 0.166 & 2.623 & $\mathrm{~B} 1.5 \mathrm{Vn}$ & -0.11 & 0.063 & 0.108 & 0.54 & 6.696 & -2.84 & 9.54 & Cep OB3 \\
\hline 900020050 & 9.82 & 0.376 & -0.042 & 0.377 & 2.686 & $\mathrm{~B} 3 \mathrm{~V}$ & -0.088 & 0.289 & 0.111 & 0.464 & 7.823 & -1.27 & 9.09 & Cep OB3 \\
\hline 900020046 & 7.41 & 0.348 & -0.073 & 0.042 & 2.609 & - & -0.121 & -0.047 & 0.082 & 0.469 & 5.393 & -3.60 & 9.00 & Cep OB3 \\
\hline 900020044 & 9.04 & 0.337 & -0.04 & 0.243 & 2.683 & B5V & -0.101 & 0.16 & 0.105 & 0.438 & 7.157 & -1.52 & 8.67 & Cep OB3 \\
\hline 900020061 & 8.14 & 0.41 & -0.096 & 0.063 & 2.612 & $\mathrm{~B} 0.5 \mathrm{~V}$ & -0.12 & -0.038 & 0.079 & 0.53 & 5.86 & -3.48 & 9.34 & Cep OB3 \\
\hline 900020047 & 7.41 & 0.287 & -0.043 & 0.071 & 2.621 & $\mathrm{~B} 1.5 \mathrm{~V}$ & -0.117 & -0.006 & 0.09 & 0.404 & 5.673 & -3.13 & 8.81 & Cep OB3 \\
\hline 900020056 & 9.33 & 0.415 & -0.07 & 0.093 & 2.633 & B1V & -0.117 & -0.008 & 0.106 & 0.532 & 7.041 & -2.91 & 9.95 & Cep OB3 \\
\hline 900020068 & 8.6 & 0.302 & -0.034 & 0.092 & 2.615 & B1V & -0.115 & 0.013 & 0.104 & 0.417 & 6.806 & -3.19 & 9.99 & Cep OB3 \\
\hline 900020069 & 7.67 & 0.355 & -0.077 & 0.05 & 2.601 & - & -0.12 & -0.04 & 0.08 & 0.475 & 5.626 & -3.76 & 9.39 & Cep OB3 \\
\hline 900020066 & 8.24 & 0.498 & -0.075 & 0.104 & 2.601 & B0IV: $\mathrm{n}$ & -0.118 & -0.013 & 0.128 & 0.616 & 5.592 & -3.64 & 9.23 & Cep OB3 \\
\hline 900020070 & 9.3 & 0.411 & -0.08 & 0.111 & 2.626 & B1V & -0.115 & 0.011 & 0.094 & 0.526 & 7.036 & -2.96 & 9.99 & Cep OB3 \\
\hline 900020076 & 7.46 & 0.368 & -0.081 & 0.008 & 2.595 & B0IV & -0.125 & -0.086 & 0.082 & 0.493 & 5.341 & -4.19 & 9.53 & Cep OB3 \\
\hline 900020075 & 7.65 & 0.493 & -0.129 & 0.07 & 2.595 & B0III & -0.121 & -0.047 & 0.074 & 0.614 & 5.009 & -3.96 & 8.97 & Cep OB3 \\
\hline
\end{tabular}




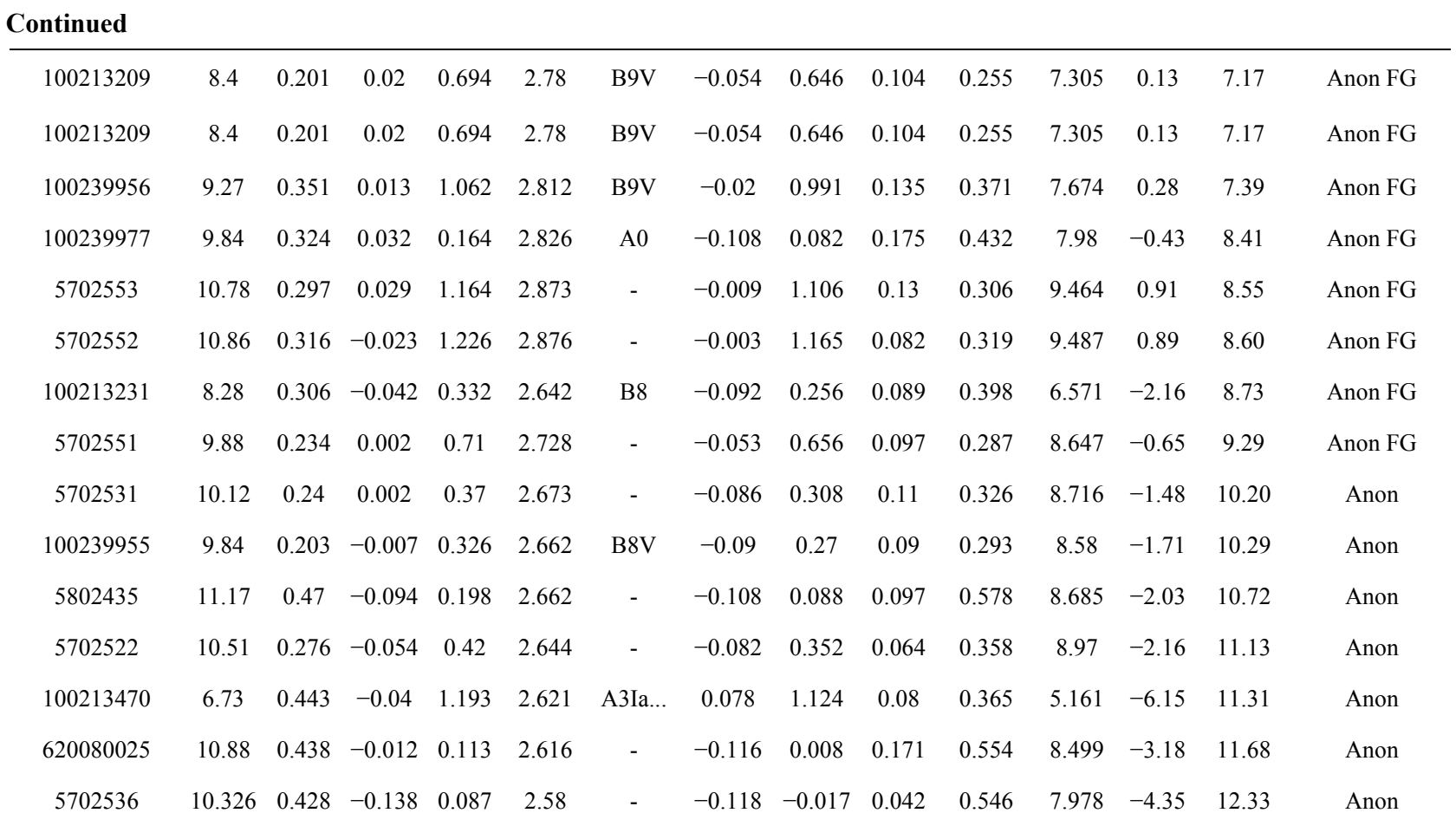

Note: The table is available in full from the authors upon request. The table includes stellar identifications (LID), $u v b y \beta$ photometric data, spectral and luminosity type, dereddened photometry and color excess, calculated absolute magnitude and true distance moduli. The portion presented here includes the stars toward the Cep OB3 association and the suggested new group at $(1, \mathrm{~b})=\left(105^{\circ}, 0.5^{\circ}\right)$ (see text for details). "FG" indicates foreground star.

photometry, calculated absolute magnitude and true distance moduli.

\section{Results}

The diagrams color excess $\mathrm{E}(\mathrm{b}-\mathrm{y})$ vs. true distance modulus (DM), dereddened visual magnitude $\mathrm{V}_{0}$ vs. dereddened color index. $(b-y)_{0}$, and $M_{V}$ vs. $(b-y)_{0}$ are presented in Figures $\mathbf{2}$ and $\mathbf{3}$ and are used to identify spatially coherent features in the studied coordinate range. Our sample is representative for several associations and young open clusters in this field. At $1=106^{\circ}$, we divide the field into two parts and consider them separately in the following analysis. The new distance and color excess estimates, as discussed below, are presented in Table 2 .

\subsection{Longitude Range $106^{\circ}-116^{\circ}$ : Cep OB3 and Cas OB2}

Cas OB2 (Rupreht 31) at average galactic coordinates (1, b) $=\left(112^{\circ}, 0.2^{\circ}\right)$ is the most spatially extended OB association previously detected within this longitude range. Several HII regions are found in the field covered by the association, Sh 2-161 and Sh 2-157 being the most prominent. The rich open clusters NGC 7510 and NGC 7654 are located within the association's boundaries. The membership to Cas OB2 has not been extensively studied. Garmany \& Stencel [16] classify Cas OB2 as a group with poorly defined main sequence (MS) and derive a true DM of 11.7 mag. Humphreys [1] provides DM = $12.1 \mathrm{mag}$, based on 16 stars. To the North of Cas OB2, the apparently very compact Cep OB3 (Rupreht 30) is located, in an area between the HII regions Sh 2-160, Sh 2-161 and Sh 2-155. Garmany \& Stencel [16] identified Cep OB3 as a group with well defined MS and, based on 29 stars, obtained a true DM of 9.3 mag. Humphreys [1] adopts true $\mathrm{DM}=9.7 \mathrm{mag}$, based on 6 stars.

The first two panels of Figure 2 present the sample stars plotted in galactic coordinates and the diagram color excess vs. true distance modulus. The locations of the three open clusters with available $u v b y \beta$ photometry present in our sample are also shown. The apparent field stars we indicate with filled symbols of different size, according to the calculated absolute magnitude. All intrinsically bright field stars $\left(\mathrm{M}_{\mathrm{V}}<-4 \mathrm{mag}\right)$ are located beyond $\mathrm{DM}=9.5$. The stars referred to Cep OB3 (open symbols) are all more reddened than $\mathrm{E}(\mathrm{b}-\mathrm{y})=0.4$ mag and very well grouped in terms of distance. There is a previously noticed group of B9-A0 foreground stars located in the direction of Cep OB3 (see Garmany \& Stencel [16] and the references therein). This group (marked with $\mathrm{x}$-symbols) is about 1 mag closer in comparison to Cep OB3. The separation evident on the $\mathrm{E}(\mathrm{b}-\mathrm{y})$ vs. DM diagram can be followed on the rest of the photometric diagrams on Figure 2. Cep OB3 has a tight MS, covering a considerable range in $\mathrm{M}_{\mathrm{V}}$. The field stars do not form 

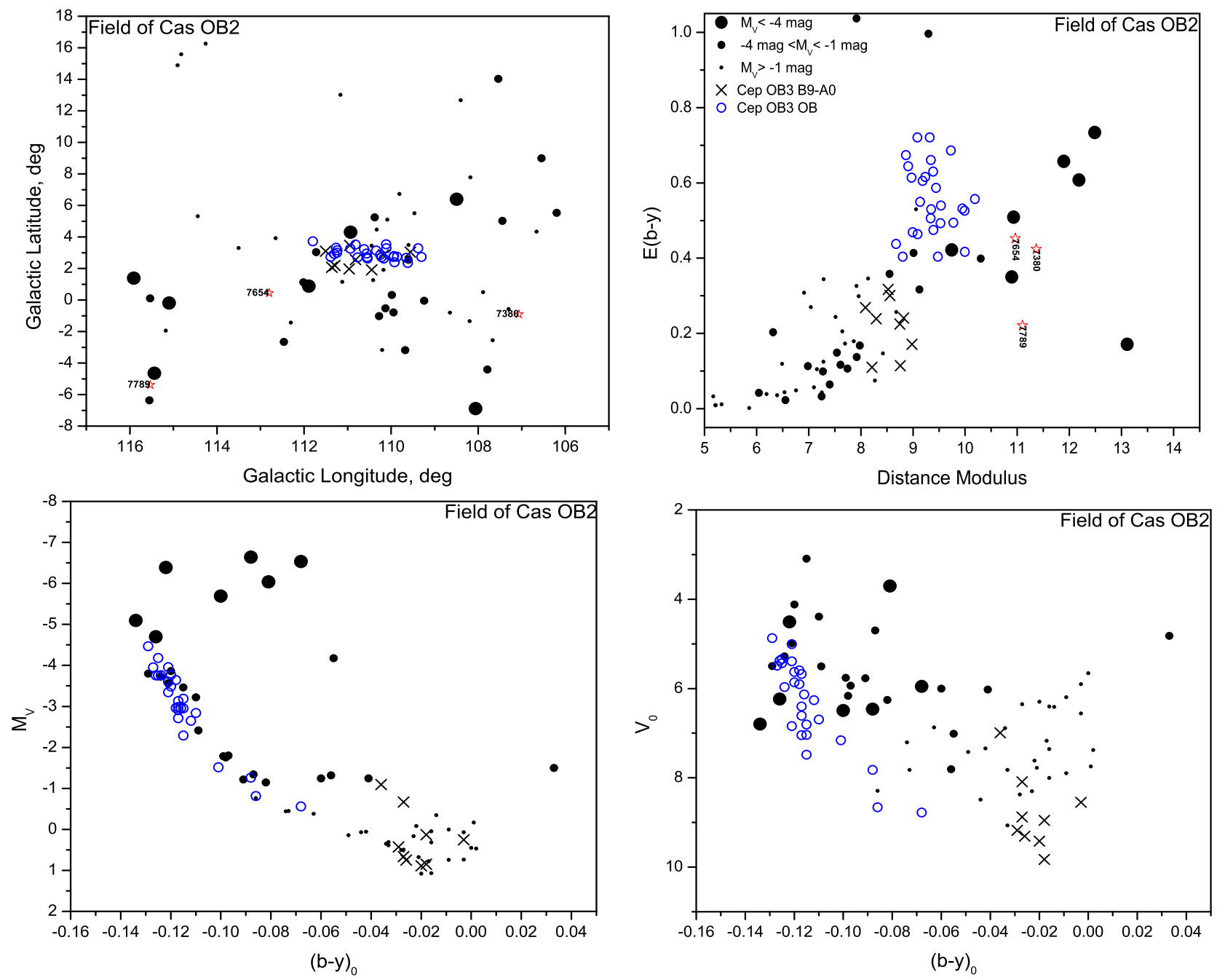

Figure 2. All O-B9 stars with calculated distances and color excesses in the sample toward the Cas OB2 and Cep OB3 associations. The diagrams color excess $E(b-y)$ vs. Distance Modulus, $V_{0} v s$. $(b-y)_{0}$ and $M_{V}$ vs. $(b-y)_{0}$ are shown. The symbols are explained in the text.

well defined main sequences, as they do not form obvious groups. The group of B9-A0 stars foreground to Cep OB3 also form a quite well defined MS on the $\mathrm{V}_{0}$ vs. (b $-\mathrm{y})_{0}$ diagram. The distance estimates to Cep OB3 existing in the literature vary between 580 and $850 \mathrm{pc}$ (see Allen et al. [17] for a recent review). The average distance, based on 27 stars, that we provide in this paper is $756 \pm 27 \mathrm{pc}(\mathrm{DM}=9.36 \mathrm{mag}$, Table 2). The O7Vn star HD 217086 (LID 900020041) is thought to be the ionizing star of Sh 2-155. We obtain a distance of $738 \mathrm{pc}$ to this star, which is twice less than the one based on the CaII HK lines (1688 pc, Megier et al. [18]). Note that the uvby $\beta$-based distance to HD 217086 is in excellent agreement with the average distance to the Cep OB3 association as obtained here.

In our sample Cas OB2 is represented by several stars intrinsically brighter than $\mathrm{M}_{\mathrm{V}}=-4$ mag. The photometric diagrams (Figure 2 bottom) indicate that these stars are evolved from the MS. They are also quite spread in distance, with an average $\mathrm{DM}=11.4$ ( \pm 1.3 s.d.) mag. All these stars are included in the previous membership lists of Cas OB2. Thus, despite of the small number of stars with $u v b y \beta$ photometry available, there are indications that the existing distance estimates for Cas OB2 maybe slightly overestimated.

The open cluster NGC 7654 is well represented in our sample (14 stars), but only the brightest stars of NGC 7789 and NGC 7380 have $u v b y \beta$ photometry presently available. We find NGC 7654 farther at about $0.3 \mathrm{mag}$ in comparison to the true DM listed in WEBDA. For NGC 7789 the true DM in WEBDA is significantly larger (up to $0.7 \mathrm{mag}$ ) in comparison to the one obtained here, and also the one provided by Dias et al. [19]. The only star from NGC 7380 with $u v b y \beta$ data available is found at a distance at agreement with the estimate for the cluster (WEBDA). Based on the available $u v b y \beta$ data, all clus- 

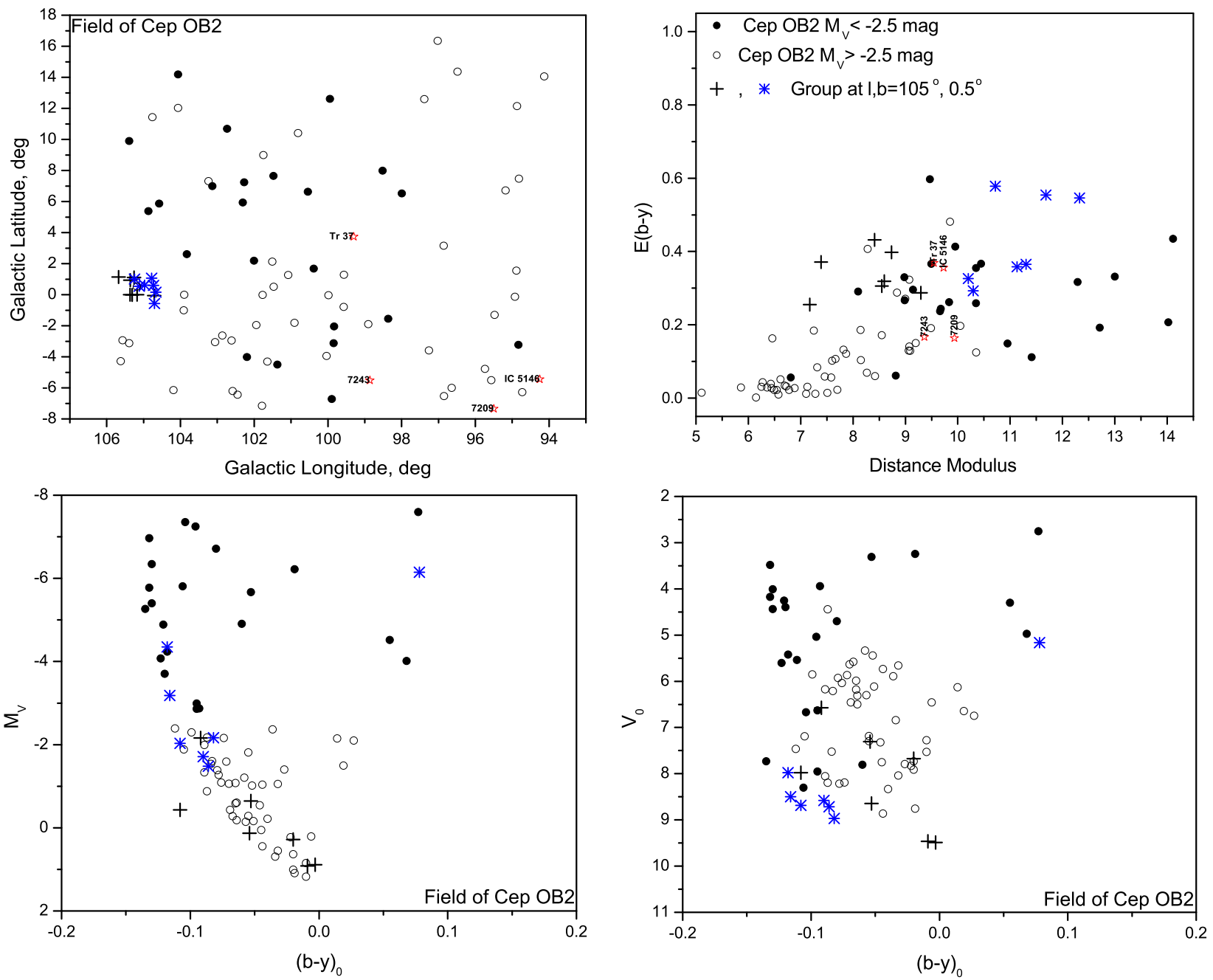

Figure 3. All O-B9 stars with calculated distances and color excesses in the sample toward the Cep OB1 and Cep OB2 associations. The diagrams color excess $E(b-y)$ vs. Distance Modulus, $V_{0} v s$. $(b-y)_{0}$ and $M_{V} v s$. $(b-y)_{0}$ are shown.

ters appear located between distance moduli $11.0 \mathrm{mag}$ and 11.4 mag. Within the errors, this range is consistent with the average distance found here for the Cas OB2 association.

\subsection{Longitude Range $94^{\circ}-106^{\circ}$ : Cep OB1 and Cep OB2}

This longitude range is dominated by the very extended Cep OB2 association (Rupreht 27). Garmany \& Stencel [16] and Humphreys [1] derive DM of 9.9 mag and 9.6 mag, respectively. Humphreys [1] found that the association extends more than $10^{\circ} \times 10^{\circ}$ in the $97^{\circ}-108^{\circ}$ longitude range and between $12^{\circ}$ and $-1^{\circ}$ galactic latitude. The open cluster Tr 37 and the prominent HII region Sh 2-131 are sometimes referred to as the core of Cep OB2. The open clusters NGC 7160, NGC 7235 and NGC 7281 are also thought to be part of the association.

Along its southern edge, Cep OB2 is adjacent to Cep
OB1. The HII region Sh 2-132 and NGC 7380 projected toward Sh 2-142 are located between the two associations. According to Humphreys [1], who lists DM $=12.7$ mag to Cep OB1, the association is about 3 times more extended along galactic longitude than in galactic latitude. Garmany \& Stencel [16] obtain DM $=12.2$ mag to Cep OB1.

The first two panels of Figure 3 present the stars considered in this subsection plotted in galactic coordinates and the diagram color excess vs. true distance modulus. The locations of the four open clusters represented to some extent in the $u v b y \beta$ sample are also indicated. We tentatively divide this subsample into two parts according to the calculated intrinsic brightness. Most of the stars intrinsically brighter than -2.5 mag (filled symbols) are located farther than $\mathrm{DM}=9.5 \mathrm{mag}$, while the rest of the stars (open circles) are foreground. Eleven of the intrinsically bright stars are found between longitudes $100^{\circ}$ to $105^{\circ}$ and latitudes $1^{\circ}$ to $8^{\circ}$, in a DM range from 9.0 to 
Table 2. Distance modulus and color excess for the clusters and $O B$ associations represented in the sample.

\begin{tabular}{cccccccccc}
\hline Name & $\mathrm{N}$ & $\mathrm{l}^{\circ}$ & $\mathrm{b}^{\circ}$ & $\mathrm{E}(\mathrm{b}-\mathrm{y})$ & s.d. & DM $($ uvby $\beta)$ & s.d. & s.e. & DM \\
\hline Cep OB3 & 27 & 110.4 & 2.9 & 0.55 & 0.09 & 9.36 & 0.39 & 0.07 & $9.3 / 9.7$ \\
Cas OB2 & 7 & 112.3 & 0.2 & 0.49 & 0.19 & 11.41 & 1.28 & 0.48 & $11.7 / 12.1$ \\
Cep OB2 & 11 & 102.3 & 5.9 & 0.34 & 0.1 & 9.76 & 0.45 & 0.15 & $9.9 / 9.6$ \\
Cep OB1 & - & - & & & - & - & & $12.2 / 12.7$ \\
Anon 105, 0.5 & 7 & 104.9 & 0.5 & 0.43 & 0.12 & 11.09 & 0.76 & 0.29 & - \\
IC 5146 & 1 & 94.25 & -5.44 & 0.36 & & 9.73 & & 9.65 \\
NGC 7209 & 2 & 95.5 & -7.34 & 0.16 & 0.02 & 9.94 & 0.47 & 19.88 & 10.33 \\
NGC 7243 & 18 & 98.86 & -5.52 & 0.17 & 0.02 & 9.36 & 0.45 & 8.52 & 9.54 \\
NGC 7380 & 1 & 107.07 & -0.9 & 0.42 & & 11.37 & & 11.73 \\
NGC 7654 & 14 & 112.8 & 0.43 & 0.45 & 0.04 & 10.97 & 0.42 & 0.11 & 10.73 \\
NGC 7789 & 3 & 115.53 & -5.38 & 0.22 & 0.02 & 11.1 & 0.52 & 0.3 & 11.27 \\
Tr 37 & 4 & 99.3 & 3.73 & 0.37 & 0.04 & 9.55 & 0.39 & 0.2 & 9.61 \\
\hline
\end{tabular}

Note: The stellar group, the number of stars on which the estimates are based, and the average galactic coordinates are shown in the first four columns. Existing distance estimates both from Garmany \& Stencel [16] and Humphreys [1] for the associations and Dias et al. [19] for the open clusters are shown in the last column. For the extinction estimates the standard deviation is provided. For all distance estimates both the standard deviation and standard error of the mean are provided.

10.5 mag. All these stars are included in the previous membership lists of Cep OB2. The average DM is 9.76 ( \pm 0.45 s.d.; \pm 0.15 s.e. $)$, consistent with previous estimates.

NGC 7243 is represented by 18 stars with $u v b y \beta$ data presently available. The average color excess and DM derived here are in good agreement with those listed in WEBDA. The $u v b y \beta$-based color excess and distance modulus of the only star from IC 5146 with such a data available are also consistent with the average parameters of the cluster (see Table 2). Of all 54 stars of NGC 7209 measured by Peña \& Peniche [20] we have classified only two stars as earlier than A0 type and calculated individual color excesses and distance moduli in agreement with the latter authors. In general, the open clusters in this subsample (Tr 37, IC 5146, NGC 7243 and NGC 7209) are all found between DM 9.4 and 10 mag.

The Cep OB1 association is represented by several intrinsically bright stars at negative latitudes, quite spread according to their $u v b y \beta$ distances. Some of them are located at DM $=12$ to $13 \mathrm{mag}$, thus consistent with previous distance estimates. The large spread in distance, however, supports previous suggestions that Cep OB1 might not be a single association (Moffat [21]). The E (b - y) vs. DM diagram on Figure 3 contains more stars farther than $11 \mathrm{mag}$, but some of them are located at positive galactic latitude. Based on our sample, we cannot reliably study the classical Cen OB1 association.

It seems possible to identify a previously undetected group of seven young stars (star-symbols) at average galactic coordinates $(1, \mathrm{~b})=\left(105^{\circ}, 0.5^{\circ}\right)$. The group is located at true $\mathrm{DM}=11.09$ ( \pm 0.76 s.d., \pm 0.29 s.e. $)$ and contains the following stars with $u v b y 98$ identifications: 5702531, 100239955, 5802435, 5702522, 620080025, 5702536 and 100213470 . These stars have similar proper motions (where available). The stars mentioned above are all more reddened than $A_{V}=0.9$ mag and are all projected near the HII regions Sh 2-138 and Sh 2-139. Several foreground stars (plus-symbols) are also located toward $(1, b)=\left(105^{\circ}, 0.5^{\circ}\right)$, all of them less massive and closer than $\mathrm{DM}=9.5 \mathrm{mag}$.

\section{Concluding Remarks}

Based on all O-B9 stars with available $u v b y \beta$ photometry in the studied longitude range we obtain homogeneous distance estimates for several stellar groups, as summarized in Table 2, and for a sample of field stars as well. In general, there is a good agreement between the $u v b y \beta$ distances and these by Garmany \& Stencel [16], which are obtained via MS fitting. However, the $u v b y \beta$ estimates, which are based on individual stellar distances should be considered more reliable for groups without well defined MS. The distance obtained here for Cep OB3 is in excellent agreement with previous estimates. There are indications that the currently adopted distance to Cas OB2 may be overestimated, but the $u v b y \beta$ sample is not large enough for firm conclusions. Although the $u v b y \beta$ sample for Cep OB2 is also relatively small, the derived distances seem to be in agreement with previous estimates. The large spread in distance for Cep OB1 suggests, how- 
ever, that this association may have a complex structure. It seems that a previously undetected group of several young stars at average galactic coordinates $(1, b)=\left(105^{\circ}\right.$, $\left.0.5^{\circ}\right)$ can be identified, projected in the vicinity of the HII regions Sh 2-138 and Sh2-139.

\section{Acknowledgements}

This work is supported by the National Science Foundation grant AST-0708950 and the University of Wisconsin Oshkosh Faculty Development Grant FDR705. This research has made use of the SIMBAD database, operated at CDS, Strasbourg, France and of the WEBDA database, operated at the Institute for Astronomy of the University of Vienna.

\section{REFERENCES}

[1] R. M. Humphreys, "Studies of Luminous Stars in Nearby Galaxies. I. Supergiants and O Stars in the Milky Way," The Astrophysical Journal Supplement, Vol. 38, 1978, pp. 309-350. http://dx.doi.org/10.1086/190559

[2] B. Strömgren, "Spectral Classification through PhotoElectric Narrow-Band Photometry," Annual Review of Astronomy and Astrophysics, Vol. 4, 1966, pp. 433-473. http://dx.doi.org/10.1146/annurev.aa.04.090166.002245

[3] D. L. Crawford and J. Mander, "Standard Stars for Photoelectric H $\beta$ Photometry," The Astronomical Journal, Vol. 71, 1966, pp. 114-118.

http://dx.doi.org/10.1086/109865

[4] N. Kaltcheva and V. Golev, "Galactic Structure toward the Carina Tangent," Publications of the Astronomical Society of the Pacific, Vol. 124, No. 912, 2012, pp. 128139. http://dx.doi.org/10.1086/664697

[5] B. Hauck and M. Mermilliod, “ $u v b y \beta$ Photoelectric Photometric Catalogue," Astronomy and Astrophysics Supplement Series, Vol. 129, No. 3, 1998, pp. 431-433. http://dx.doi.org/10.1051/aas:1998195

[6] J. E. Gaustad, P. R. McCullough, W. Rosing and D. Van Buren, "A Robotic Wide-Angle H $\alpha$ Survey of the Southern Sky," Publications of the Astronomical Society of the Pacific, Vol. 113, No. 789, 2001, pp. 1326-1348. http://dx.doi.org/10.1086/323969

[7] T. McGlynn, K. Scollick and N. White, "SkyView: The Multi-Wavelength Sky on the Internet," Proceedings of the 179th Symposium of the International Astronomical Union, Baltimore, 26-30 August 1996, pp. 465-466.

[8] D. L. Crawford, "Empirical Calibrations of the $u v b y \beta$ Systems. II. The B-Type Stars," Astronomical Journal, Vol. 83, No. 1, 1978, pp. 48-63. http://dx.doi.org/10.1086/112176

[9] D. Kilkenny and D. C. B. Whittet, "On the Intrinsic $u v b y$
Colours of Early-Type Supergiants," Monthly Notices of the Royal Astronomical Society, Vol. 216, 1985, pp. 127137.

[10] L. A. Balona and R. R. Shobbrook, "A Re-Calibration of the Luminosities of Early-Type Stars-Its Effect on the Cepheid Luminosity Scale," Monthly Notices of the Royal Astronomical Society, Vol. 211, 1984, pp. 375-390.

[11] N. T. Kaltcheva and R. W. Hilditch, "The Distribution of Bright OB Stars in the Canis Major-Puppis-Vela Region of the Milky Way," Monthly Notices of the Royal Astronomical Society, Vol. 312, No. 4, 2000, pp. 753-768. http://dx.doi.org/10.1046/j.1365-8711.2000.03170.x

[12] N. Kaltcheva and V. Makarov, "The Structure and the Distance of Collinder 121 from Hipparcos and Photometry: Resolving the Discrepancy," The Astrophysical Journal Letters, Vol. 667, No. 2, 2007, pp. L155-L157. http://dx.doi.org/10.1086/522495

[13] N. Kaltcheva and J. Knude, "A Test of B-Type Star $H \beta$ Photometric Distances via the HIPPARCOS Parallaxes," Astronomy and Astrophysics, Vol. 337, 1998, pp. 178182.

[14] J. Torra, D. Fernández and F. Figueras, "Kinematics of Young Stars. I. Local Irregularities," Astronomy and Astrophysics, Vol. 359, 2000, pp. 82-102.

[15] N. Kaltcheva and V. Golev, "Improved Distances to Several Galactic OB Associations," Stellar Clusters and Associations: A RIA Workshop on GAIA, Granada, 23-27 May 2011, pp. 299-303.

http://sca.iaa.es/content/proceedings

[16] C. D. Garmany and R. E. Stencel, "Galactic OB Associations in the Northern Milky Way Galaxy. I-Longitudes $55^{\circ}$ to $150^{\circ}, "$ Astronomy and Astrophysics Supplement Series, Vol. 94, No. 2, 1992, pp. 211-244.

[17] T. S. Allen, R. A. Gutermuth, E. Kryukova, S. T. Megeath, J. L. Pipher, et al., "Spitzer Imaging of the Nearby Rich Young Cluster, Cep OB3b," The Astrophysical Journal, Vol. 750, No. 2, 2012, p. 125. http://dx.doi.org/10.1088/0004-637X/750/2/125

[18] A. Megier, A., A. Strobel, G. A. Galazutdinov and J. Krełowski, "The Interstellar Ca II Distance Scale," Astronomy and Astrophysics, Vol. 507, No. 2, 2009, pp. 833-840. http://dx.doi.org/10.1051/0004-6361/20079144

[19] W. S. Dias, B. S. Alessi, A. Moitinho and J. R. Lépine, "New Catalogue of Optically Visible Open Clusters and Candidates," EAS Publications Series, Vol. 10, 2003, p. 195. http://dx.doi.org/10.1051/eas:2003148

[20] J. H. Peña and R. Peniche, "Uvby- $\beta$ Photometry of Open Clusters. IV. NGC 1444, NGC 1662, NGC 2129, NGC 2169, and NGC 7209," Revista Mexicana de Astronomia y Astrofisica, Vol. 28, No. 2, 1994, pp. 139-152.

[21] A. F. J. Moffat, "Photometry and Structure of the Young Open Cluster NGC 7380," Astronomy and Astrophysics, Vol. 13, 1971, pp. 30-39. 